\title{
Cerebellar infarct in a patient struck by lightning: a case report
}

\author{
Bitoti Chattopadhyay ${ }^{1}$, Atri Chatterjee ${ }^{1}$, Pijush Kanti Biswas ${ }^{2}$, \\ Nirod Baran Debnath ${ }^{3}$ \\ ${ }^{1}$ Junior resident, Department of Medicine, Nil Ratan Sircar Medical College, Kolkata, India. \\ ${ }^{2}$ Assistant Professor, Department of Medicine, Nil Ratan Sircar Medical College, Kolkata, India. \\ ${ }^{3}$ Professor, Department of Medicine, Nil Ratan Sircar Medical College, Kolkata, India.
}

\begin{abstract}
Lightning strike can result in varied neurologic consequences ranging from hypoxic encephalopathy to intracranial hemorrhage. However, lightning strike resulting in cerebellar stroke is very rare. We present the case of a patient who developed cerebellar ataxia following a lightning strike. Magnetic resonance imaging revealed infarcts in both cerebellar hemispheres. The patient recovered with minimal disabilities with standard treatment of ischemic stroke. To the best of our knowledge, this is the second report of such an association and highlights the relatively complete functional recovery of patients in a very rare situation.
\end{abstract}

Keywords: Cerebellar ataxia, cerebellar infarct, lightning.

\section{Introduction}

Most victims of a lightning strike have multisystem involvement $[1,2]$. However, injuries to the cardiovascular and central nervous system are most significant. Several case reports have presented various neurological complications following a lightning strike, but very few of them relate to cerebellar ataxia. Among the other known consequences of such an event, cognitive dysfunction, with mood disorders, is most common [2]. More serious effects like basal ganglia bleeds have been frequently reported [3, 4]. There are reports of spinal cord injury leading to paraplegia or quadriplegia [5]. Literature search reveals a single reported case of multiple cerebellar infarcts in a patient of lightning strike, who recovered after conservative treatment [1]. Here, we report a case of lightning strike resulting in acute onset cerebellar ataxia due to multiple infarcts in both cerebellar hemispheres.

\section{Case report}

A 24 year old man was brought to the emergency room with altered sensorium six hours after being struck by lightning in his farm. On arrival, his Glasgow coma scale (GCS) score was 13/15 (E3 M6 V4), pulse $100 /$ minute, regular, blood pressure 120/68 mm Hg, respiratory rate 22/min and $\mathrm{SpO}_{2}$ of $94 \%$ in room air. Neurological examination showed bilateral symmetric and reactive pupils, flexor plantar response. All deep tendon jerks were depressed. However, there was no paresis, cranial nerve palsy or nystagmus. The patient's electrocardiogram was normal. His complete blood count, electrolytes, liver and renal function tests were also within normal limit. Serum creatine phosphokinase was raised (416 U/L) with normal creatine kinase muscle band fraction (12 U/L). Computed tomography (CT) of the brain showed mild cerebral oedema.

On the fifth day of hospital stay, the patient was fully conscious but developed ataxia with left sided intention tremor. Examination revealed scanning speech, dysmetria and dysdiadochokinesia more readily demonstrable on the left side. Still there was no nystagmus. At this point, the patient was afebrile, cardiac examination did not reveal any murmur and there was no sign of any vasculitic phenomenon.

Magnetic resonance imaging of brain obtained on the same day showed infarcts involving both cerebellar hemispheres, predominantly on the left side (Fig 1). However, magnetic resonance angiography was normal. Transthoracic echocardiography was normal and was followed up with an echocardiography with injection of agitated saline infusion to exclude a persistent foramen ovale. Doppler study of the carotid arteries, as well as abdominal aorta and its branches did not reveal any abnormality.

The patient's coagulation profile, which included prothrombin time, activated partial thromboplastin time, thrombin time and serum fibrinogen, was normal. Serological investigations for the hepatitis B virus surface antigen, anti-hepatitis $\mathrm{C}$ virus antibody, human immune-deficiency virus and anti-nuclear antibody were negative. The patient was started on $150 \mathrm{mg} /$ day of aspirin along with other supportive measures. The patient was discharged on 12th day of admission and after six months of follow up, he is doing well.

\section{Conclusion}

About $20 \%$ of people who are struck by lightning are killed, rest survive with various degrees of disability [6]. Although rare, serious lightning injuries can cause permanent neurological damage. Immediate 
neurologic manifestations in survivors include altered consciousness, impaired vision, hearing loss, seizures and paralysis [7].

In our patient, possible causes of acute onset altered sensorium and cerebellar signs included stroke. The initial CT brain revealed cerebral edema without any focal lesion. Nevertheless, these data could not explain the development of ataxia in the victim. This necessitated a follow up MRI, which identified infarcts involving cerebellar hemispheres. This phenomenon can be explained by direct electrolytic effect of the electrical charge passing through the brain, heating effect of the electrical current and mechanical trauma of the lightning stroke. To exclude common causes of stroke in young, additional investigations were done, which were all within the normal limit.

In conclusion, we report a unique case of bilateral cerebellar infarcts in a patient of lightning strike. To the best of our knowledge, this is the second report of such an association in literature.

\section{Figure Legend}

Figure 1. Magnetic resonance imaging (T1 FLAIR sequence) showed bilateral cerebellar infarcts (arrowheads).

\section{References}

[1] S. Aslan, S. Yilmaz, O. Karcioglu. Lightning: an unusual cause of cerebellar infarction. Emerg Med J 2004; 21 : 750-51.

[2] T. J. Janus, J. Barrash. Neurologic and neurobehavioral effects of electric and lightning injuries. J Burn Care Rehabil 1996; 17:40915 .

[3] B. Ozgun, M. Castillo. Basal ganglia hemorrhage related to lightning strike. Am J Neuroradiol 1995;16: 1370-71.

[4] A. Aggarwal A, P. Salunke. Bolt from the blue: Basal ganglion bleed following lightning strike. Surg Neurol Intl 2011; 2: 170.

[5] H.H. Tan, S. H. Goh. Lightning injuries: Changi Hospital experience. Hong Kong J Emerg Med 2003; 10: $223-32$.

[6] M. A. Cooper, C. J. Andrews, R. L. Holle, et al. Lightning injuries. In: Auerbach P,ed. Wilderness medicine. 4th ed. St Louis: Mosby, 2001.

[7] M. A. Cooper, C. J. Andrews. Lightning injuries. In: Auerbach PS, Geehr ED, eds. The management of wilderness and environmental injuries. 3rd ed. St Louis: Mosby Year Book, 1995.

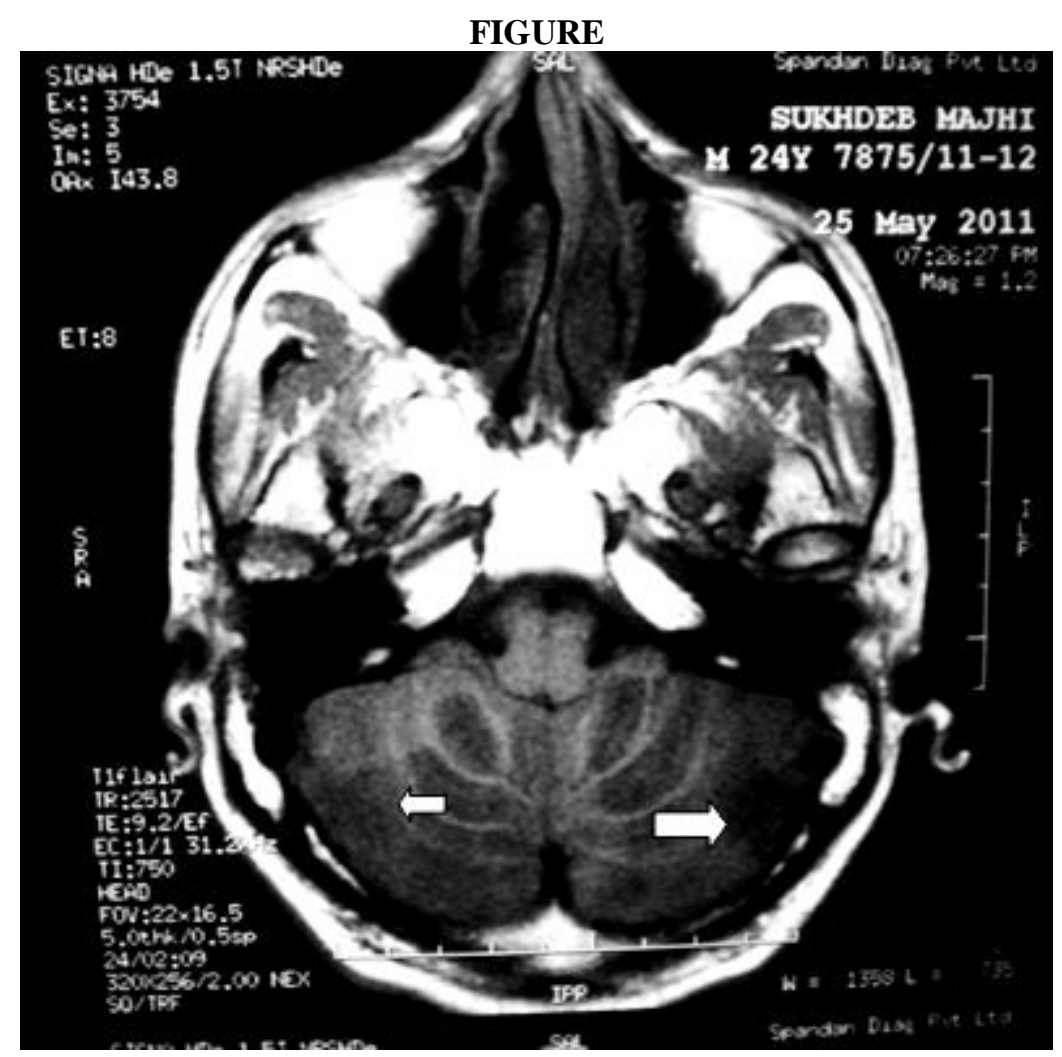

